\title{
Corrosion Residue Debris Left by Implant Materials to the Patient's Body
}

\author{
Gulsen Akdogan ${ }^{1 *}$ and Mohammad Ghaith Almasry \\ Department of Biomedical Engineering, Erciyes University, Turkey \\ *Corresponding author: Gulsen Akdogan, Department of Biomedical Engineering, Erciyes University, Turkey \\ To Cite This Article: Gulsen Akdogan.Corrosion Residue Debris Left by Implant Materials to the Patient's Body. Am J Biomed Sci \& Res. 2019 - 6(3). \\ AJBSR.MS.ID.001022. DOI: 10.34297/AJBSR.2019.06.001022.
}

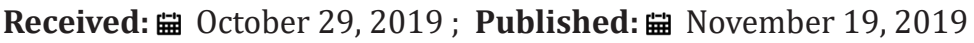

\begin{abstract}
In the field of biomaterial science, the corrosion of metallic implants in the body cannot be avoided so the selection of biocompatible and corrosion resistant materials is so important, whereas the corrosion can decrease the lifetime of implants. Human body represents a very aggressive corrosive environment to metallic implants. Although some pure or commonly known alloys as implants can be used in combination with the body when used alone, they can cause problems if used in places where material pair is used without observing the electrochemical potential difference between them. As a result, electrochemical corrosion will happen. A Battery formation due to electrochemical potential difference between materials and subsequent electron transfer increases the amount of debris that breaks from metal in the body environment. Debris formation and their effects on the human body are the initial reason for shortening the duration of implant leading to implant failure and of course revision operations.
\end{abstract}

Keywords: Biomaterials; Biocorrosion; Metal corrosion; Implants; Debris; Aseptic loosening

\section{Introduction}

Today, due to the continuous development of health services, increasing life expectancy, increasing population rate, demographic and non-demographic changes in all age groups, obesity and many other factors, a higher rate of recurrent joint implants is expected in the future [1]. According to the literature, it is stated that the use of joint implants will increase in the coming years [2]. In the US alone, more than 1 million joints fail each year after a maximum of 15 to 25 years of use. Repeated revision surgery is performed for replacement of failed joint implants [3]. Biomaterials must be selected according to strict criteria whereas we have so many reasons lead to implant failure in the human body. One of the most important reasons is corrosion and it's one of the most challenging problems. By the time corrosion's debris lead to aseptic loosening and implant failure. In this article we are going to discuss corrosion of metallic implants and battery formation which is triggered when using different metals or alloy components in addition to how the developed debris lead to aseptic loosening and implant failure.

\section{Process of Corrosion}

Corrosion can be defined as the undesirable chemical reaction of the metal with the environment, starting with electron transfer between metals leading to degradation of metallic materials which can lead to loss the function of implant $[4,5]$. The human body environment presents too aggressive environment to the implants because it contains water, dissolved oxygen, proteins, chloride, hydroxide, various anions such as phosphate and chloride ions and cations like $\mathrm{Na}+\mathrm{Ca} 2+[6]$. So, it plays the role of electrolyte fluid between alloy components or between the different metals which can be used in knee or hip implants parts to trigger electrochemical corrosion and battery formation. Even some materials known to be perfectly compatible with the body may initiate battery formation if the potential difference between them is not respected where they are used as a pair of materials. For example, as found in the alloys used on the tibial and femoral sides of the total knee implant sections $[7,8]$. Therefore, the formation of a battery and electron transfer triggering between metal surfaces as shown as in Figure 1.

The hostile environment provides adverse and aggressive conditions to the metals used for implantation and we can say that metals undergo brutal electrochemical corrosion in the body [9]. Here it is important to mention the effect of surface oxide film on metallic corrosion process. This film plays a very important role 
to prevent further corrosion after reaching equilibrium across the surfaces. Disequilibrium can be occurred by many reasons for example, binding of some proteins which can be found in surrounding environment to the surface of implant and many other reactions between the film and environment. After disequilibrium process the regeneration time of this thin film is a very important factor to determine the number of released ions due to interaction between metal surface and surrounding tissues. The process of metal ions leaching is going to continue until the oxide film healing again. The regeneration time depends on the metallic components and the implant environment and plays a major role in corrosion processes and debris [6]. Oxide film surface's regeneration time for some alloys is given in Table 1 [10]. According to the Table 1 in stainless steel 316L alloys regeneration time is the longest, so the released metallic ions number is the highest comparing to the others.

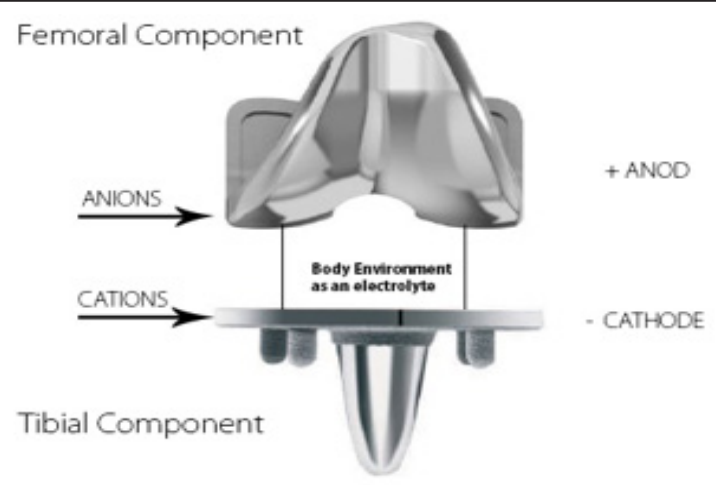

Figure 1: A drawing showing the electrochemical corrosion between the components of the knee implant.

Table 1: Regeneration time of surface oxide film [10].

\begin{tabular}{|c|c|}
\hline Alloy & Regeneration Time \\
\hline SS $316 \mathrm{~L}$ & $35.3 \mathrm{~min}$ \\
\hline $\mathrm{Zr}-2.5 \mathrm{Nb}$ & $13.8 \mathrm{~min}$ \\
\hline Co- $28 \mathrm{Cr}-6 \mathrm{Mo}$ & $12.7 \mathrm{~min}$ \\
\hline Ti-6Al-4V & $8.2 \mathrm{~min}$ \\
\hline
\end{tabular}

\section{Corrosion and Implant Failure}

Corrosion of metallic implants is a slow progressive procedure and occurs in continuous way because of the interactions between the implants and body environment. Corrosion of metallic implants and related debris is one of the most important reasons to implant failure. It has been found that $42 \%$ of metallic implant failures had been happened due to corrosion and erosion-corrosion. This ration needs to be considered [11,12]. The formation of debris residues by corrosions resulting from the potential difference between alloy elements composed of more than one metal in the human body is one of the reasons for the failure of the implants by initiating slow progressive inflammation at the bone implant interface. This local and inflammatory deceptive condition called inflammation; mainly due to the interaction of implant debris with cells of the immune system [3]. Aseptic loosening is a serious long-term complication of revision surgery due to clinical / surgical risk [13]. The corrosion, and related debris reduce the life expectancy of the implant much more than expected whereas debris of the implants and the biological reactions resulting from the debris have been clearly identified as a primary cause of long-term implant failure [14].

\section{Conclusion}

Corrosion of metallic implants in the human body, battery formation and subsequent electron transfer increase the amount of debris from the metal to the body environment which can be accumulate in the bone- implant interface and initiate slow progressive inflammation leading to aseptic loosening, failure and revision surgery. Whereas the corrosion cannot be avoided in the human body, corrosion and its prevention still a very important field of research.

\section{References}

1. Culliford D, Maskell J, Judge A, Cooper C, Prieto-Alhambra D, et al. (2015) "Future Projections of Total Hip and Knee Arthroplasty in the UK: Results from The UK Clinical Practice Research Datalink", Osteoarthritis and Cartilage 23(4): 594-600.

2. Pabinger C, Lothaller A, Portner C, Geissler X (2018) “Projections of Hip Arthroplasty in OECD Countries Up To 2050". HIP International 1-9.

3. Hallab N, Jacobs O (2017) "Chemokines Associated with Pathologic Responses to Orthopedic Implant Debris", Frontiers in Endocrinology 8 (5).

4. Park J, Lakes S (2007) Biomaterials an Introduction $3^{\text {rd }}$ ed. New York, USA, Springer Science and Business Media LLC.

5. Cadosch D, Chan E, P Gautschi O, Filgueira L (2009) Metal is not inert: Role of metal ions released by biocorrosion in aseptic loosening-Current concepts. Journal of Biomedical Materials Research Part A 91(4):12531262.

6. Manivasagam G, Dhinasekaran U, Rajamanickam K (2010) Biomedical Implants: Corrosion and Its Prevention - A Review". Recent Patents on Corrosion Science 2: 40-54

7. Dipankar C, Ay CH, Rajshree M (2014) Wear Debris Characterization and Corresponding Biological Response: Artificial Hip and Knee Joints Materials 7(2): 980-1016.

8. Akdogan G (1999) Investigation of Characteristics of Biocorrosion and Biocompatibility of Ti-6Al-4V Alloys. Gazi University, Ankara, Turkey p. 44-48.

9. NS Manam, WSW Harun, DNA Shri, SAC Ghani, T Kurniawan, et al. (2017) Study of corrosion in biocompatible metals for implants: A review. Journal of Alloys and Compounds 701(15): 698-715.

10. Hanawa T (2003) Reconstruction and Regeneration of Surface Oxide Film on Metallic Materials in Biological Environments. Corrosion Reviews 21: 2-3.

11. Hansen D (2008) Metal Corrosion in the Human Body: The Ultimate BioCorrosion Scenario. The Electrochemical Society Interface 31-34.

12. Aksakal B, Yildirim OS, Gul H (2004) Metallurgical Failure Analysis of Various Implant Materials Used in Orthopedic Applications. Journal of Failure Analysis and Prevention 4(3): 19-23.

13. Kurtz SM, Ong KL, Schmier J, Zhao K, Mowat F, et al. (2009) Primary and Revision Arthroplasty Surgery Caseloads in The United States From 1990 To 2004. J Arthroplasty 24 (2): 195-203. 
14. Robertsson O, Knutson K, Lewold S, Lidgren L (2001) The Swedish Knee Arthroplasty Register 1975-1997: An Update with Special Emphasis
On 41,223 Knees Operated on in 1988-1997. Acta Orthop Scand 72 (5): 503-513. 